\title{
Morphological Studies on Optically Anisotropic Rodlike Textures of Poly(butylene terephthalate)
}

\author{
Chie Sawatari, Takashi MuranaKa, ${ }^{*}$ and Masaru Matsuo** \\ Department of Clothing Science, Faculty of Home Economics, \\ Nara Women's University, Nara 630, Japan
}

(Received June 24, 1982)

\begin{abstract}
An interesting phenomenon is reported on the formation of rodlike textures of poly(butylene terephthalate) (PBT). When an amorphous film and a film having spherulitic textures were annealed under pressure, rodlike textures arranged randomly were formed. They were observed by means of polarizing microscopy and light scattering measurement. Moreover, for an elongation of the film for which no superstructure was observed by polarizing microscopy, the rodlike textures were oriented at a particular angle with respect to the stretching direction and formed networks.

KEY WORDS Poly(butylene terephthalate) / Spherulitic Texture / Rodlike Texture / Particular Angle / Networks /
\end{abstract}

Because of its moldability and very high crystallization rate, poly(butylene terephthalate) (PBT) has important applications as an engineering thermoplastics. ${ }^{1}$ For instance, it is used as a component in block copolymers, where it confers rubber elasticity by introducing soft segments. ${ }^{2-4}$ These attractive properties indicate the need for studying the structure, crystallization rates, and melting behavior of this polymer.

Gilbert and Hybart ${ }^{5,6}$ studied the crystallization rates and morphology in terms of the effect of lengthening the aliphatic component and of having odd and even numbers of carbon atoms in it by preparing polymers with various variations in the chemical structure. Their results showed that lower extents of crystallization were observed for the polymer containing the shorter sections of aliphatic units. This is contradictory to the prediction that polymers with longer sections would be expected to lead to more flexible chains and to crystallize more readily. The melting points of the polymers were reported to decrease considerably on increasing the length of the aliphatic sequence between the aromatic rings in the chains. More quantitative results on similar polymers were reported by Pratt and Hobbs $^{7}$ using differential scanning calorimetry

* Kogaku Co., Ltd., Osaka.

** To whom all correspondence should be addressed.
(DSC) and depolarization microscopy.

The morphology of PBT has been examined to some extent. Spherulites of PBT show a Maltese cross at $45^{\circ}$ to the crossed polars under a polarizing microscope. The lobes of the $H_{\mathrm{v}}$ light scattering patterns have intensity maxima at azimuthal angles of $0,90,180$, and $270^{\circ}$. This characteristic pattern is due to the scattering from spherulites whose optical axes lie at an angle of approximately $45^{\circ}$ to the spherulite radius. These spherulites were named as "unusual" spherulites by Stein and Misra,${ }^{8}$ in comparison with the "usual" spherulites showing a Maltese cross along the polar direction under a polarizing microscope. According to the theoretical prediction, this type of pattern arises from two dimensional spherulites whose optical axes do not twist randomly about the radius but lie on the disk plane. ${ }^{9}$ Further investigations of the usual and unusual spherulites were carried out in detail by means of electron microscopy. ${ }^{10}$

This paper is concerned with the formation of rodlike textures of PBT and their deformation. The results of this investigation indicate a new need for studying the structure and properties of PBT; here they are analyzed by means of light scattering, polarizing microscopy, and infrared dichroism and 
the results are discussed in connection with crystallinity and differential thermal analysis (DTA) data.

\section{EXPERIMENTAL}

Crude pellets of PBT were treated with ethanol for $30 \mathrm{~h}$ in a Soxhlet's extractor before their use as specimens. The intrinsic viscosity of the extracted pellets in $o$-chlorophenol at $25^{\circ} \mathrm{C}$ was $[\eta]=0.635$. According to the method of Stein and Misra, the extracted pellets were sandwiched between Teflon sheets at $245^{\circ} \mathrm{C}$ for $10 \mathrm{~min},{ }^{8}$ at a pressure of $140 \mathrm{~kg}$ $\mathrm{cm}^{-2}$. The molten sample was quenched by being plunged into an ice-water bath to obtain amorphous specimens. The film thickness was about $50 \mu \mathrm{m}$. The weight percent crystallinity of these "amorphous" samples was measured by a pycnometer using a carbon tetrachloride : $n$-heptane mixture as a medium was found to be $7 \%$, assuming densities of crystalline and amorphous forms as $1.396^{11}$ and $1.282,{ }^{8}$ respectively.

As a result of many observations, it became clear that one obtains rodlike textures in PBT in deformed state. Amorphous films obtained by the above method were sandwiched between Teflon sheets again and annealed in the press at $140 \mathrm{~kg}$ $\mathrm{cm}^{-2}$, at $200^{\circ} \mathrm{C}$ for several minutes as described below. The resulting film was quenched by being plunged into an ice-water bath. Samples prepared in this fashion exhibited a characteristic X-type light scattering pattern under the $H_{v}$ polarization condition, suggesting the existence of rodlike textures. The film obtained in this manner is labelled as specimen $\mathrm{A}$ in this paper.

For comparison purposes, another sample was prepared. The extracted pellets were sandwiched between Teflon sheets and molded in the press at $140 \mathrm{~kg} \mathrm{~cm}^{-2}$, at $245^{\circ} \mathrm{C}$ for $10 \mathrm{~min}$. The molten sample was quenched by being plunged into water at $50^{\circ} \mathrm{C}$. The unusual spherulites with a $45^{\circ}$ Maltese cross were observed in the film prepared by the above method. This film was then annealed and quenched under the same conditions as for specimen A. The obtained film is labelled as specimen B.

The amorphous film was held at temperatures between 80 and $200^{\circ} \mathrm{C}$ and stretched to various desired extension ratios. In this process, the formation of rodlike textures was observed by using polarizing microscopy and light scattering. The experiments indicated above show that it is rather complicated to obtain rodlike textures in nondeformed samples but it is easy to obtain them by elongation processes. The experiments are described in more detail below.

Light scattering patterns were obtained with a $3 \mathrm{~mW} \mathrm{He}-\mathrm{Ne}$ gas laser as a light source. Diffuse surface scatter was avoided by sandwiching the specimen between microscope cover slides with silicone oil of an appropriate refractive index as an immersion fluid. Polarizing microscopy was done using a Nikon OPTIPHOT-POL (XTP-11). This made visible structures which were too small to be detected by light scattering. Melting thermograms were measured using the Shimadzu Differential Thermal Analyzer (DT-20B) with a heating rate of $20^{\circ} \mathrm{C} \mathrm{min}^{-1}$. A $5 \mathrm{mg}$ sample of each film was cut into fine pieces and placed in standard aluminium sample pans.

Incidentally, wide angle $\mathrm{X}$-ray diffraction patterns from all undrawn specimens used in this experiment showed circular rings characterizing random orientation of PBT crystallites when the Xray beam was directed parallel to the film surface. This result does not indicate the planer orientation of benzene rings parallel to the film surface.

\section{RESULTS AND DISCUSSION}

Figure 1(a) shows a polarizing micrograph of the "amorphous" film. Indistinct superstructures are observed. They are neither the usual or unusual spherulites; no Maltese cross is observed. The superstructures are so small that they can not be detected by $H_{v}$ light scattering; the pattern is shown in Figure 1(b).

Figure 2(a) shows a polarizing micrograph of specimen $\mathrm{A}$ and (b) in enlargement. It is seen that the internal structures are not typical of a spherulitic structure but rather composed of more or less randomly arranged optically anisotropic rodlike textures. The micrographs indicate the existence of two types of rods; they show negative and positive birefringence using a $1 / 4$ wave plate. The number of rods showing negative birefringence was more than that showing positive birefringence. The former indicates the orientation of chain axes nearly perpendicular to the rod axis and the latter indicates a nearly parallel orientation. Figures 2(c) and (d) show light scattering patterns under $H_{\mathrm{v}}$ and $V_{\mathrm{v}}$ polarization conditions, respectively. The $H_{\mathrm{v}}$ pat- 


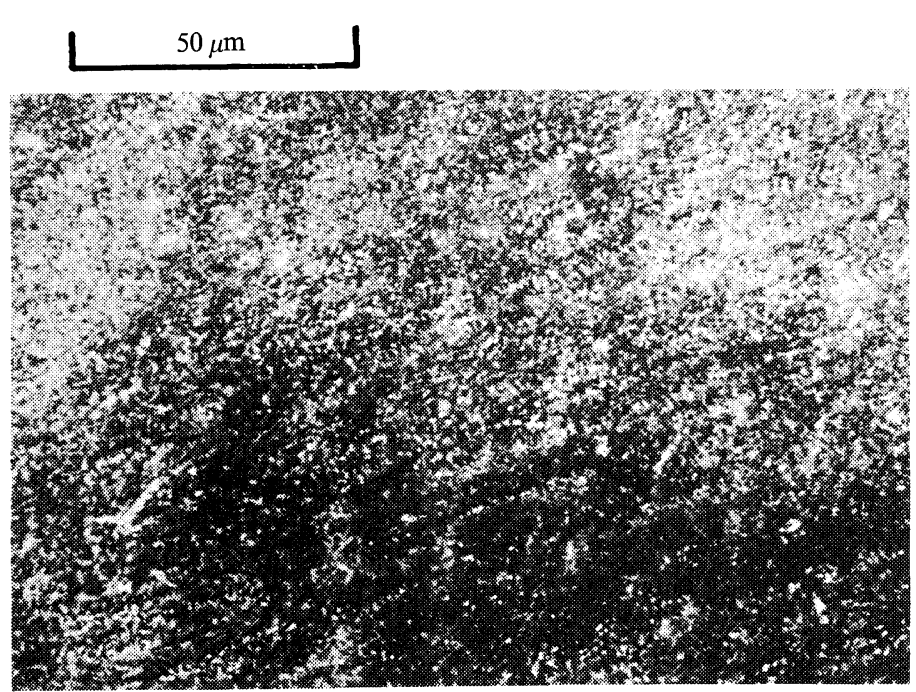

(a)

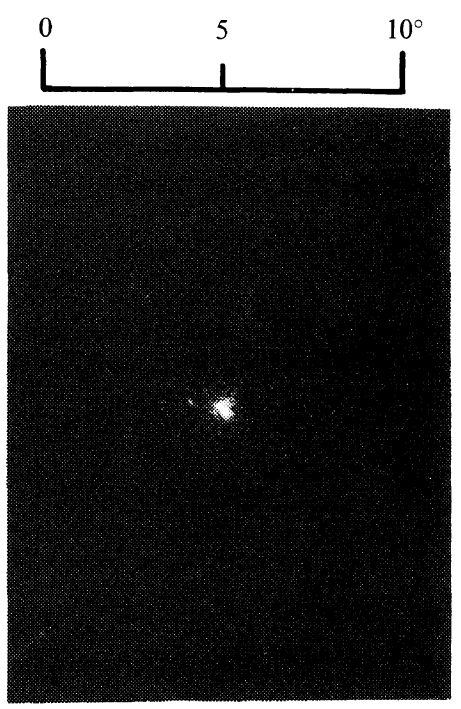

(b)

Figure 1. Structure of amorphous PBT film prepared by molding. (a) polarizing micrograph; (b) $H_{\mathrm{v}}$ light scattering pattern.

tern has four lobes which show no indication of the intensity maxima along the scattering angle which are characteristic of a spherulitic scattering pattern. The intensity is greatest at the scattering center and decreases monotonically with increasing scattering angle. This is indicative of the presence of rodlike textures as has been previously reported by Rhodes and Stein. ${ }^{12)}$ The azimuthal dependence of the pattern arising from the rods is determined by the value of the angle $\omega$ between the optical axis and the long axis of the rods. The $H_{\mathrm{v}}$ scattering pattern indicates that the optical axis direction within the rod is either along $\left(\omega=0^{\circ}\right)$ or perpendicular $(\omega=$ $90^{\circ}$ ) to the long axis of the rod. The lobes of the $V_{\mathrm{v}}$ scattering pattern are extended in the meridian direction, which indicates the existence of rods whose optical axes are oriented nearly perpendicular to the direction of the rod axes. The result of the two scattering patterns is in good agreement with the observation of the polarizing micrographs that the number of the rods having negative birefringence is greater than the number having positive birefringence.

Figures 3(a) and (b) show a polarized light micrograph and an $H_{\mathrm{v}}$ light scattering pattern from a specimen which was prepared by sandwiching the amorphous film between Teflon sheets at $200^{\circ} \mathrm{C}$ for $10 \mathrm{~min}$ with no pressure before quenching into ice water. Indistinct structures were observed with polarizing microscopy but they were not detected by $H_{\mathrm{v}}$ light scattering. In this method of sample preparation, we do not observe the presence of rodlike textures as shown in Figure 2.

Considering the results in Figures 2 and 3, we can draw an interesting conclusion; the amorphous films must be held under pressure to prepare specimens having rodlike textures. In order to provide more conclusive evidence for this conclusion, another experiment was carried out. That is, specimen B was prepared from a specimen having the unusual spherulitic structures as shown in Figure 4. The $H_{\mathrm{v}}$ and $V_{\mathrm{v}}$ patterns in this figure have the same shape observed by Stein and Misra. ${ }^{8}$ Under the polarizing microscope using a $1 / 4$ wave plate, two types of spherulites were observed, having thier optical axes either at $+45^{\circ}$ or $-45^{\circ}$ to the spherulitic radius. The probabilities for the optical axis to be $+45^{\circ}$ or $-45^{\circ}$ were confirmed to be equal, as discussed by Stein and Misra. ${ }^{8}$

Figure 5(a) shows an $H_{\mathrm{v}}$ light scattering pattern from the resulting sample $B$. It is the superposition of a four-leaf clover type pattern and a small X-type pattern. The X-type is enlarged in Figure 5(c). The intensity is greatest in the center and decreases monotonically with increasing scattering angle; this indicates the presence of rodlike textures. As can be 


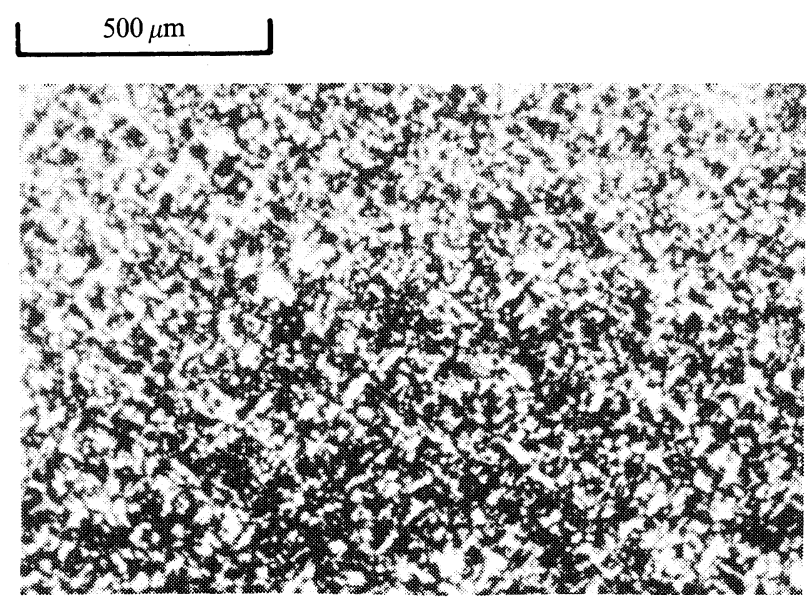

(a)

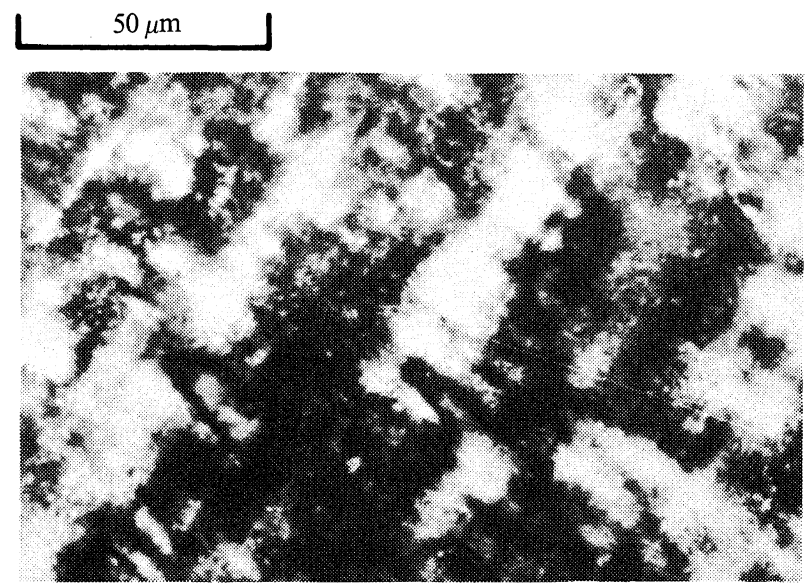

(b)

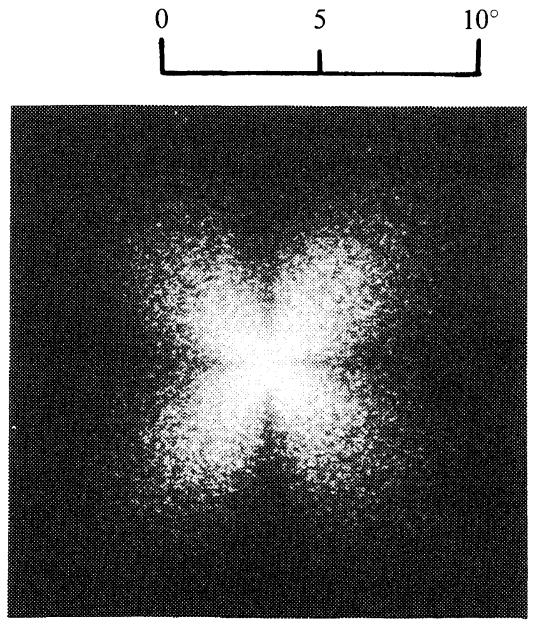

(c)

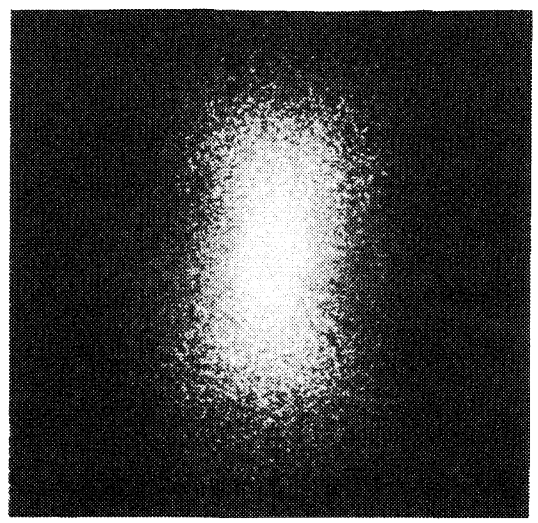

(d)

Figure 2. Structure of specimen A. (a) polarizing micrograph; (b) enlargement of (a); (c) $H_{\mathrm{v}}$ light scattering pattern; (d) $V_{\mathrm{v}}$ light scattering pattern.

seen in Figure 5(d), the coexisrence of spherulitic and rodlike textures in the polarizing microscope by introducing a $1 / 4$ wave plate was clearly observed and the spherulites are enlarged in Figure 5(e). The rods looked like the indistinct whiting in the polarized micrographs. Moreover, we found that the two kinds of rods, with positive and negative birefringence coexist in the film. The number of negative rods is greater than the number of positive ones. The size of the rods was larger than those in Figure 2. The presence of the rods was impossible to confirm using the $V_{\mathrm{v}}$ light scattering because of the strong intensity of the incident beam around the scattering center. The number of spherulites was not increased through the annealing process used to prepare specimen B. The rods were formed by the transformation of an isotropic medium leaving the spherulites surrounded by the rods instead of the medium.

Figures 6(a) and (b) show the crystallinity for specimens $\mathrm{A}$ and $\mathrm{B}$ as a function of annealing time at $200^{\circ} \mathrm{C}$. The crystallinity shows a considerable increase in the first $5 \mathrm{~min}$ and then tends to level off beyond $5 \mathrm{~min}$. This agrees with prior observations 


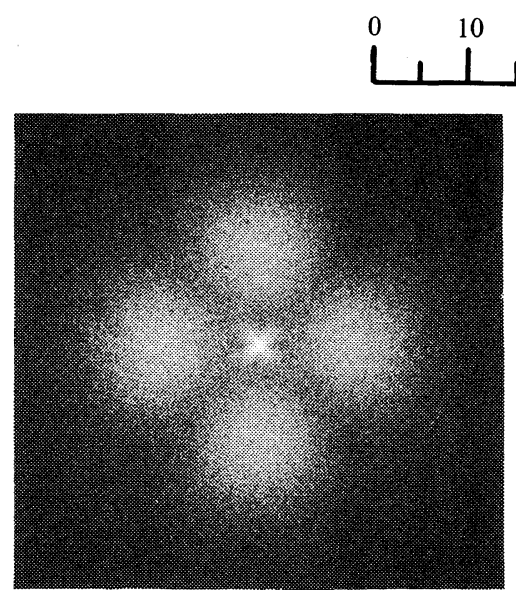

(a)
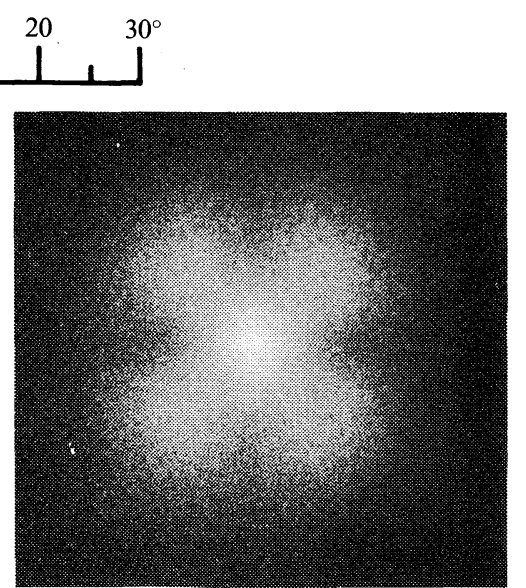

(b)

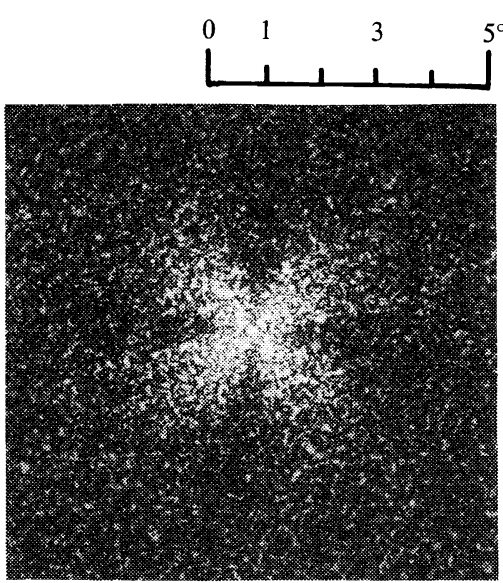

(c)

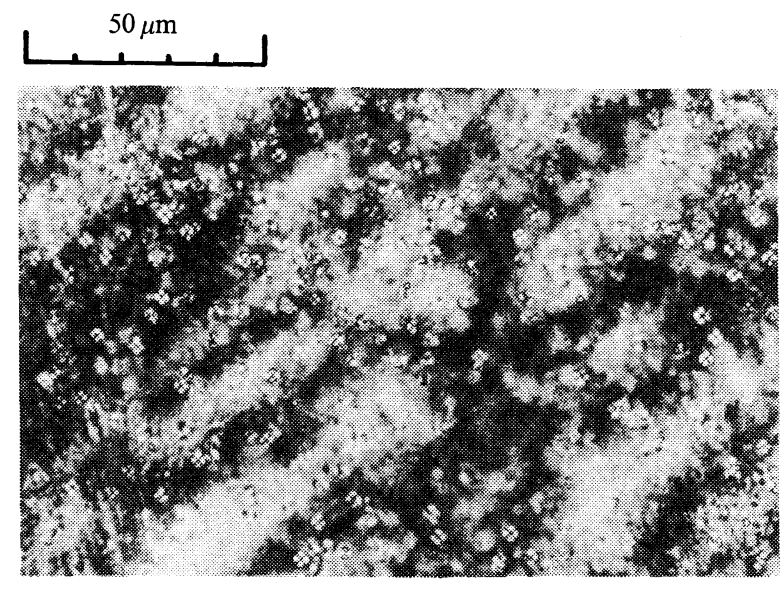

(d)

\section{$\longleftarrow^{10 \mu \mathrm{m}}$}

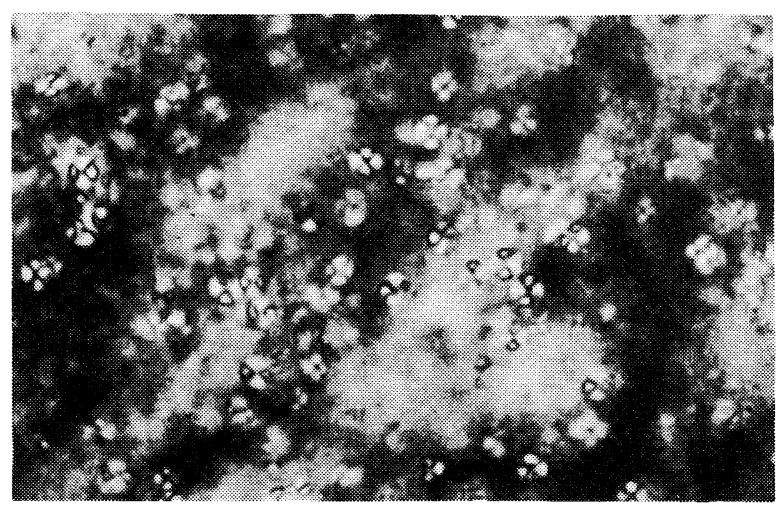

(e)

Figure 5. Structure of specimen B. (a) $H_{\mathrm{v}}$ light scattering pattern; (b) $V_{\mathrm{v}}$ light scattering pattern; (c) micrograph of spherulites. 


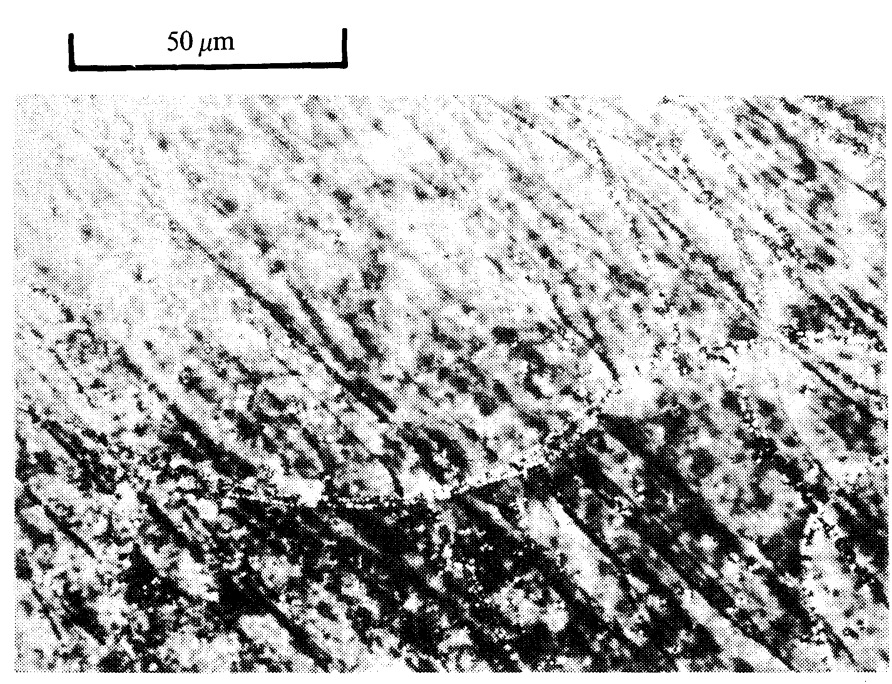

(a)

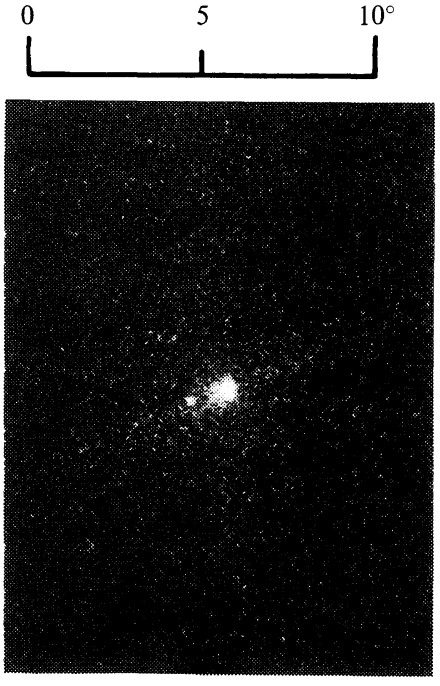

(b)

Figure 3. Structure of specimen prepared by sandwiching an amorphous film at $200^{\circ} \mathrm{C}$ for $10 \mathrm{~min}$ without any pressure. (a) polarizing micrograph; (b) $H_{\mathrm{v}}$ light scattering pattern.

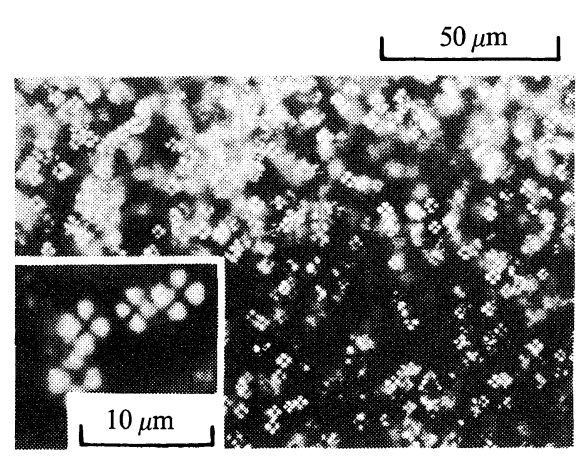

(a)

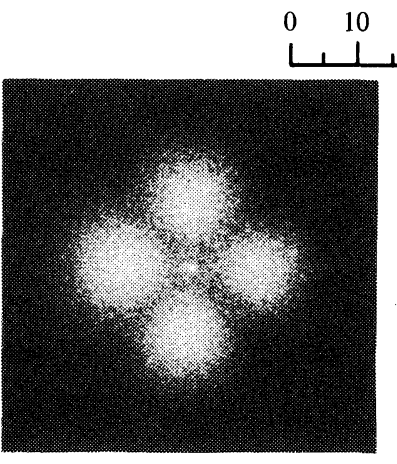

(b)

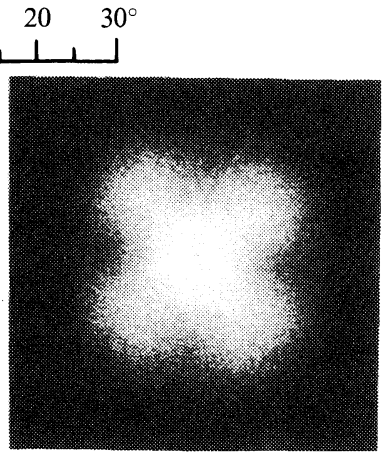

(c)

Figure 4. Morphology of unusual spherulites. (a) polarizing micrograph; (b) $H_{\mathrm{v}}$ light scattering pattern, (c) $V_{\mathrm{v}}$ light scattering pattern.

that PBT crystallizes much more rapidly than polyethylene terephthalate. ${ }^{5}$ The intrinsic viscosity $[\eta]$ did not decrease through the annealing process for samples A and B. However, $[\eta]$ decreases when the annealing was carried out without any pressure, in which we suggest marked lowering of the molecular weight of PBT is due to oxidization. The increase in crystallinity was found to be essentially independent of factors influencing the formation of the rodlike textures by a subsequent experiment.

Figure 7 shows polarizing micrographs of speci- mens prepared by annealing the "amorphous" samples at three different temperatures, (a) $50^{\circ} \mathrm{C}$, (b), $30^{\circ} \mathrm{C}$, and (c) $20^{\circ} \mathrm{C}$ with a constant annealing time of $5 \mathrm{~min}$ in the press at $140 \mathrm{~kg} \mathrm{~cm}^{-2}$. The crystallinities of the specimen annealed at 50 and $20^{\circ} \mathrm{C}$ are about 20 and $8 \%$, respectively. The size of the rodlike textures becomes larger with decreasing annealing temperature. The crystallinities and the size were not changed in spite of an increase of annealing time beyond $5 \mathrm{~min}$ and they were affected only by annealing temperature. When the above 


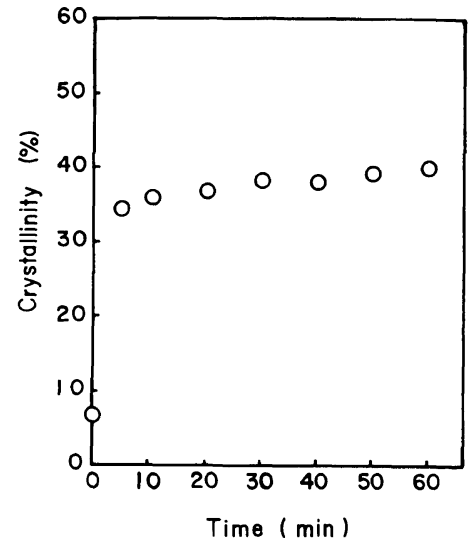

(a)

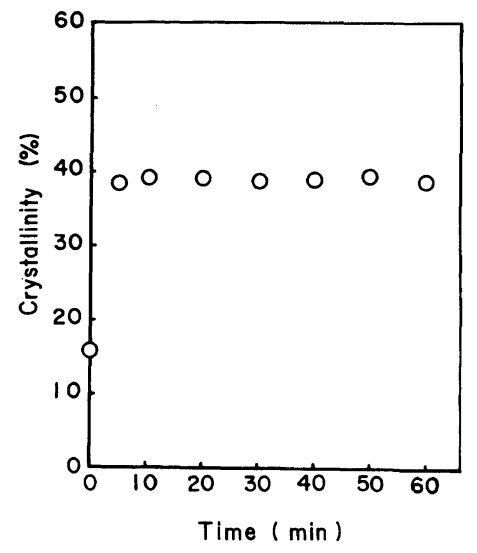

(b)

Figure 6. Change in crystallinity with the increase of annealing time at $200^{\circ} \mathrm{C}$. (a) specimen $\mathrm{A}$; (b) specimen B.

experiments were carried out with no pressure, we can not observe any rodlike texture. This result indicates that the formation of rodlike textures requires a pressure to be applied to amorphous films during the annealing process. This experimental result is interesting, but the cause is not yet resolved.

Figure 8 shows DTA melting thermograms for the amorphous film (curve (a)) and two kinds of specimen A prepared with different annealing times (curve (b); annealed for $5 \mathrm{~min}$ and curve (c); annealed for $40 \mathrm{~min}$ at $200^{\circ} \mathrm{C}$ ). As can be seen in these curves, the appearance and its ultimate coalescence with the final melting peak at $220^{\circ} \mathrm{C}$ of an additional low temperature peak was affected by the annealing time. The shape of curve (a) was also obtained for the specimen having spherulitic texture shown in Figure 4. The shapes of curves (b) and (c) were the same for two kinds of specimen $B$ that were also prepared by annealing at $200^{\circ} \mathrm{C}$ for 5 and $40 \mathrm{~min}$, respectively. The above results indicate that the shape of the DTA trace is independent of the appearance of superstructures as well as their shape and that it is only dependent on the annealing time. We also note that even though the DTA curves differ, the crystallinity is approximately the same for samples annealed for 5 and $40 \mathrm{~min}$ as shown in Figure 6.

Based on the series of experiments discussed above, we conclude that annealing under high pressure is necessary to obtain randomly arranged rodlike textures. However, the rodlike textures may be formed easily through the elongation of amorphous films. The specimens were preheated for $15 \mathrm{~min}$ at the chosen temperature. The elongation was carefully done to avoid macroscopic necking; this was actually possible around up to an extension ratio of $\lambda=2.0$ at temperatures between 80 and $200^{\circ} \mathrm{C}$, but the film exhibited a clear necking beyond $\lambda=2.5$. The film remained transparent through the elongation but started to whiten slightly in the necking region.

Figure 9 shows polarizing micrographs of the films stretched at $120^{\circ} \mathrm{C}$. As shown in photograph (a), no superstructure was observed in this preheated film prior to deformation, although the crystallinity increased from 7 to $18 \%$ through the preheating for $15 \mathrm{~min}$. This indicates, as discussed already by Stein and $\mathrm{Misra}^{8}{ }^{8}$ that during crystallization from the glassy state, the nucleation density is very high, which results in a superstructure of very small dimensions that cannot be resolved by the polarizing microscope. The photographs from (b) to (f) seem to suggest rodlike textures existing in the interior of the specimen. The rodlike textures appeared at draw ratios at low as $\lambda=1.2$.

The rods are oriented at a particular angle with respect to the stretching direction and form networks. These network structures are quite similar to those observed for linear polyethylene prepared by a calendar molding process. ${ }^{13,14}$ That is, we consider that these units correspond to single rownucleated cylindrites or an assembly of them. The 


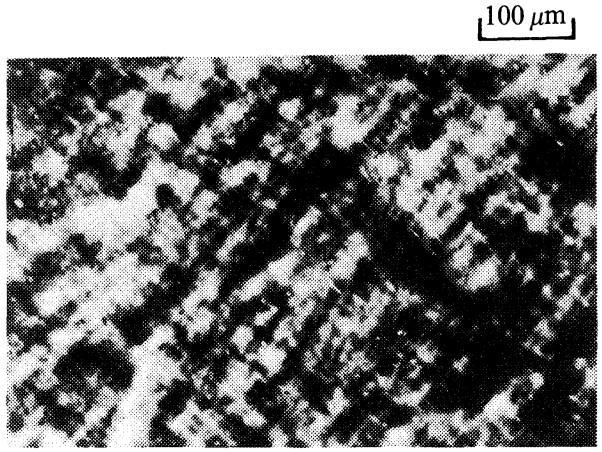

(a)

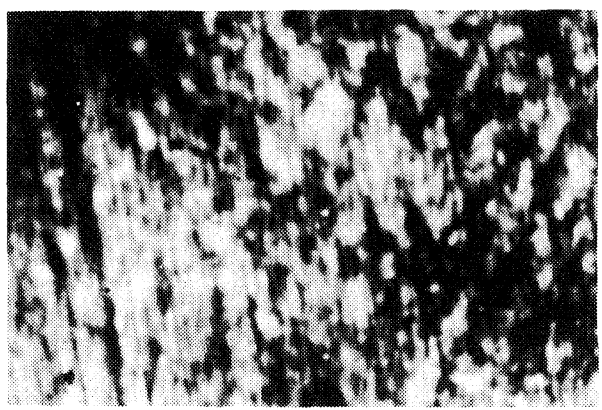

(b)

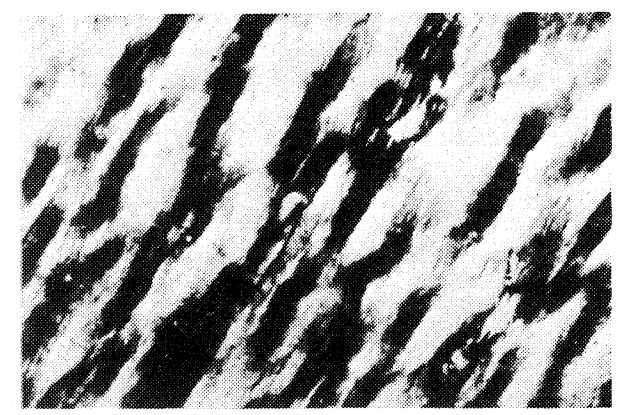

(c)

Figure 7. Polarizing micrographs of specimen after annealing the "amorphous" samples at different annealing temperatures. (a) $50^{\circ} \mathrm{C}$; (b) $30^{\circ} \mathrm{C}$; (c) $20^{\circ} \mathrm{C}$.

boundaries between the adjacent crystallites become a part of an amorphous phase having zero birefringence. The rod axes at $\lambda=1.2$ were predominantly oriented perpendicular to the stretching direction rather than parallel, which indicates the predominant growth of the rods in the direction perpendicular to the stretching direction. The crystallinity in this film was increased only slightly during the formation of the rodlike textures from the isotropic medium, as shown in Figure 10. The

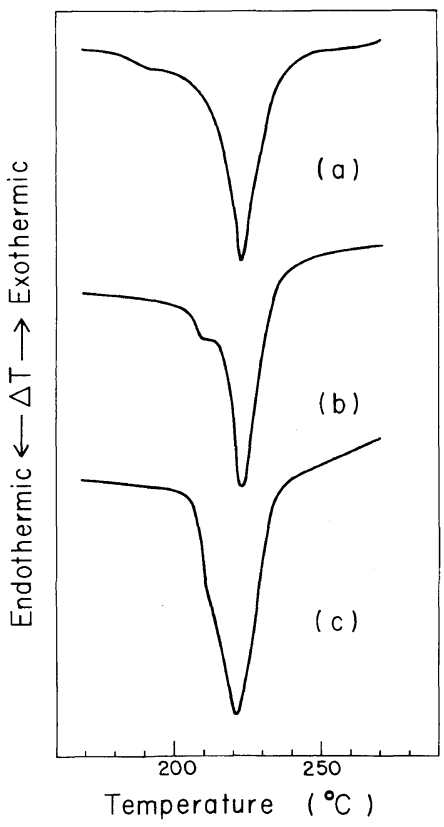

Figure 8. DTA melting thermograms.

increase in crystallinity is almost independent of elongation of the specimens and is only affected by the temperature of annealing. With further increase in elongation, the rods become oriented more and more in the stretching direction while preserving the network structure. This identification is rather clear, which indicates continuity or integrity of rods with elongations. This tendency is quite different from that for linear polyethylene. ${ }^{13}$ The light scattering technique was used to study the chain orientation of the rodlike textures and the network in detail.

Figure 11 shows the change in $H_{\mathrm{v}}$ scattering patterns with increasing extension ratio $\lambda$. The scattering up to $\lambda=3.0$ shows the superposition of a broad four-leaf pattern as lobes and a sharp fourleaf pattern as streaks. The angle $\alpha$ that the $H_{v}$ scattering lobes make with respect to the equator is apparently related to the angle that the optically anisotropic rodlike textures make with respect to the stretching direction. Therefore, from $H_{v}$ scattering patterns, we can show that the rods are oriented at a particular angle with respect to the stretching direction. With elongation more than $\lambda=3.5$, the four-leaf pattern becomes indistinct, which indicates a disruptive deformation of the rodlike textures. The sharp four-leaf streaks have sometimes been observed for oriented crystalline films 
Anisotropic Rodlike Textures of Poly(butylene terephthalate)

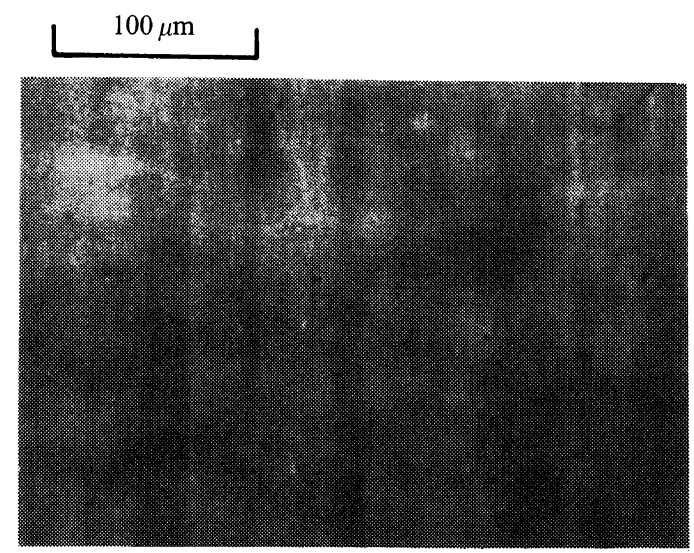

(a) $\lambda=1.0$

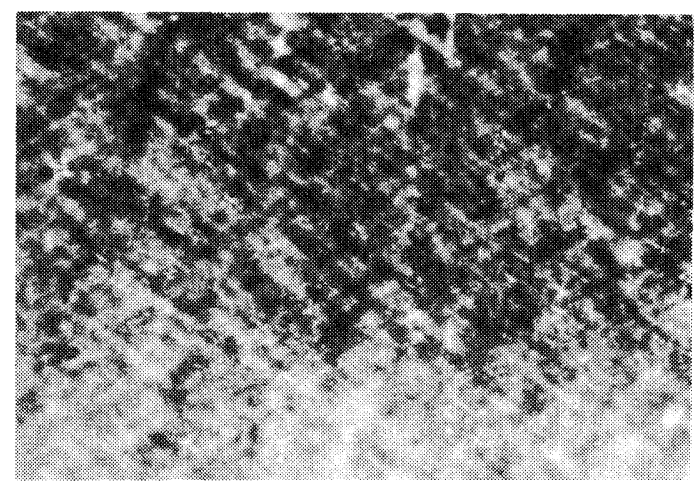

(b) $\lambda=1.2$

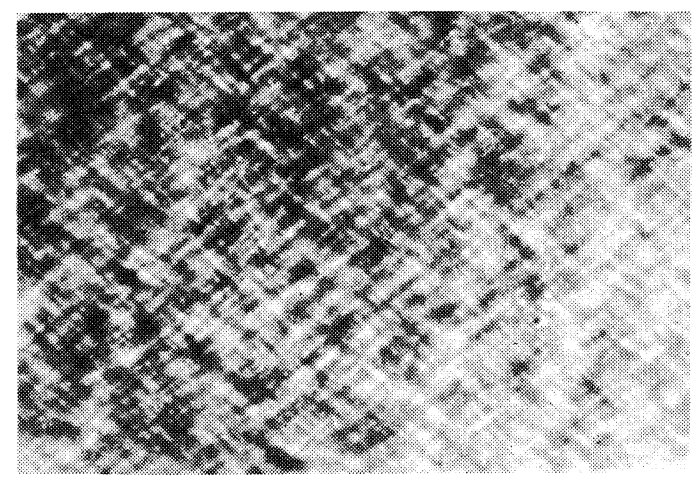

(c) $\lambda=1.5$

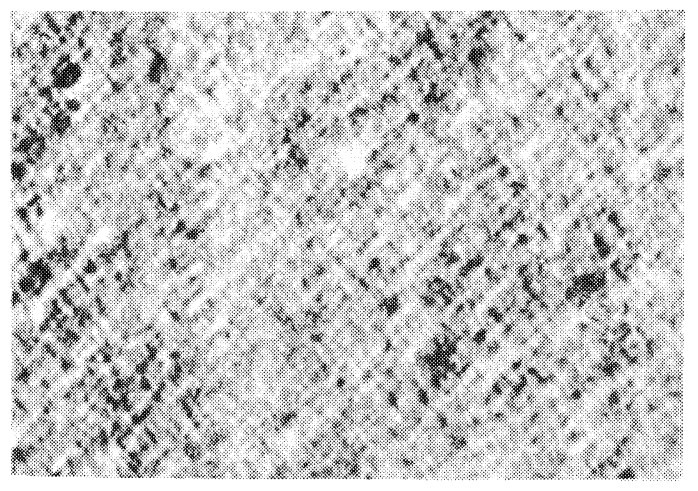

(d) $\lambda=2.0$

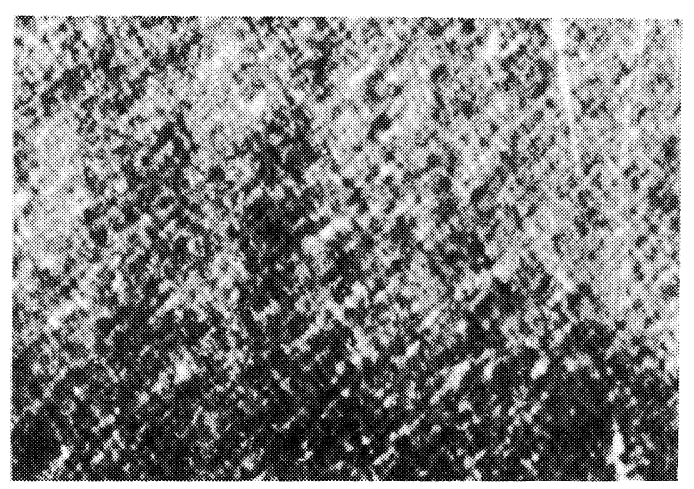

(e) $\lambda=2.5$

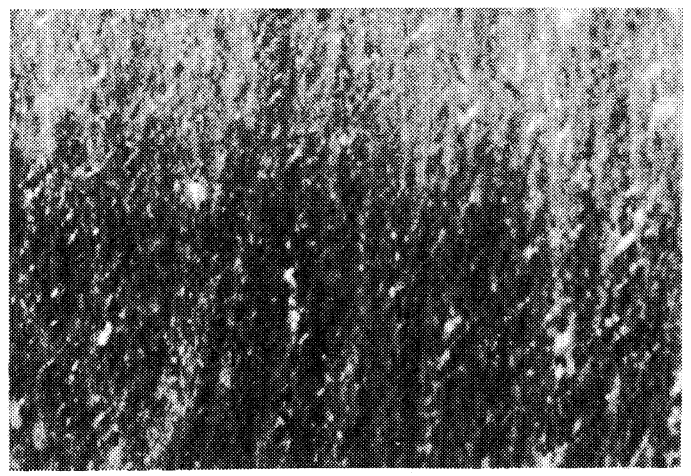

(f) $\lambda=3.5$

Figure 9. Polarized micrographs of PBT films stretched at $120^{\circ} \mathrm{C}$.

such as tubular-extended poly(1-butene $)^{15}$ and polyethylene terephthalate. ${ }^{16}$ The streaks in those cases have been analyzed as scattering from an assembly of sheaves. Accordingly, we believe that the streaks in Figure 11 reflect interparticle interference effects from the rods. Therefore, the angle $\gamma$ that the sharp $H_{\mathrm{v}}$ scattering streaks make with respect to the equator is thought to be associated 


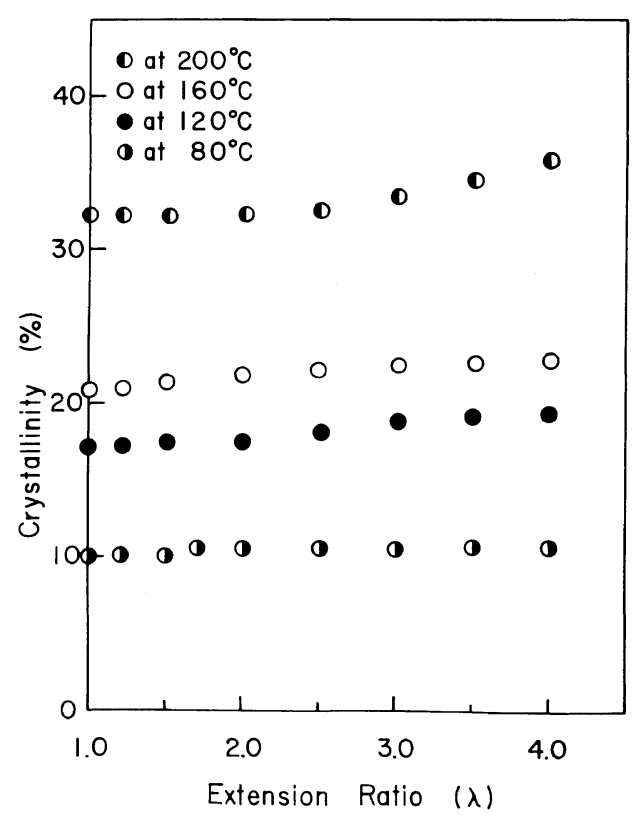

Figure 10. Crystallinity $v s$. extension ratio of PBT film stretched at $80,120,160$, and $200^{\circ} \mathrm{C}$.

with an average orientation angle between the line connecting the center of the rods and the stretching direction. The above presumption concerning the angles $\alpha$ and $\gamma$ as shown in Figure 12 was confirmed as indicated in the schematic diagrams in the case of $\lambda=1.5$. The white lines in the polarizing micrograph (d) are drawn along assemblies of rods to make clear the interparticle interference effect. Actually, as shown in Figure 12, the angles $\alpha$ and $\gamma$ are associated with an average orientation angle of the rods and that of their assembly, respectively, with respect to the stretching direction.

The polarizing micrographs and light scattering patterns for the specimens stretched at $80,120,160$, and $200^{\circ} \mathrm{C}$ were essentially the same and consequently essentially identical rodlike textures were formed and deformed in these films, despite the differences in crystallinity shown in Figure 10. The orientation of the optical axes within the rodlike textures was quantitatively postulated by observing the change in the $H_{\mathrm{v}}$ scattering pattern upon rotation of the stretching direction of the specimen, with respect to the polarization direction. The intensity distribution becomes maximum, when the angle between polarizer and stretching direction is $45^{\circ}$ and this tendency becomes considerable with increasing $\lambda$. This result indicates that the optical axes are predominantly oriented in the stretching direction with increase of $\lambda$.

The orientation behavior may be determined, in detail, by measuring the change in birefringence as shown in Figure 13. The increase indicates the preferential orientation of the optical axes, i.e., the molecular chains of PBT. The birefringence quantities are almost the same despite the differences of crystallinities and the elongation temperatures. This tendency is quite similar to the results obtained from polarizing microscope and light scattering.

The orientation behavior of PBT molecules can also be discussed in terms of the second order orientation factor of the transition moment of the $j$ th group as measured by infrared dichroism. The orientation factor is defined as

$$
F_{20}^{j}=\frac{3\left\langle\cos ^{2} \alpha_{j}\right\rangle-1}{2}=\frac{A_{\|}^{j}-A_{\perp}^{j}}{A_{\|}^{j}+2 A_{\perp}^{j}}
$$

where $\alpha_{j}$ is the orientation angle of the transition moment of the $j$-th group with respect to the stretching direction and $A_{\|}^{j}$ and $A_{\perp}^{j}$ are optical densities using base-line method at the absorption maximum of polarized light parallel and perpendicular to the stretching direction, respectively. Figure 14 shows the results calculated using eq 1 for specimens stretched at $160^{\circ} \mathrm{C}$. The orientation behavior of the transition moment of the $v(\mathrm{C}=\mathrm{O})$ band whose absorption occurs at $3460 \mathrm{~cm}^{-1}$ gives almost random orientation at $\lambda=1.2$ but change to negative orientation with further elongation. This absorption corresponds to the overtone of a fundamental vibration occurring at $1716 \mathrm{~cm}^{-1}$. The specimens used in this experiment were somewhat thick with the optical densities $A_{\|}^{j}$ and $A_{\perp}^{j}$ of the fundamental vibration being 1.7 ; it was impossible to obtain the orientation factor using the fundamental vibration. The transition moment of the $v(\mathrm{C}=\mathrm{O})$ band may be assumed to orient perpendicular to the chain direction. If this is the case, the above orientation behavior suggests a highly positive orientation of the PET molecules. The orientation behavior of the transition moment of the $\omega\left(\mathrm{CH}_{2}\right)$ band, the wagging vibration of the $\mathrm{CH}_{2}$ group, shows a considerable increase of positive, orientation with increasing $\lambda$. This transition moment may be assumed to be oriented parallel to the chain axes. This contrasting behavior between the $\omega\left(\mathrm{CH}_{2}\right)$ and $v(\mathrm{C}=\mathrm{O})$ vibrations can be simply understood as a 
Anisotropic Rodlike Textures of Poly(butylene terephthalate)
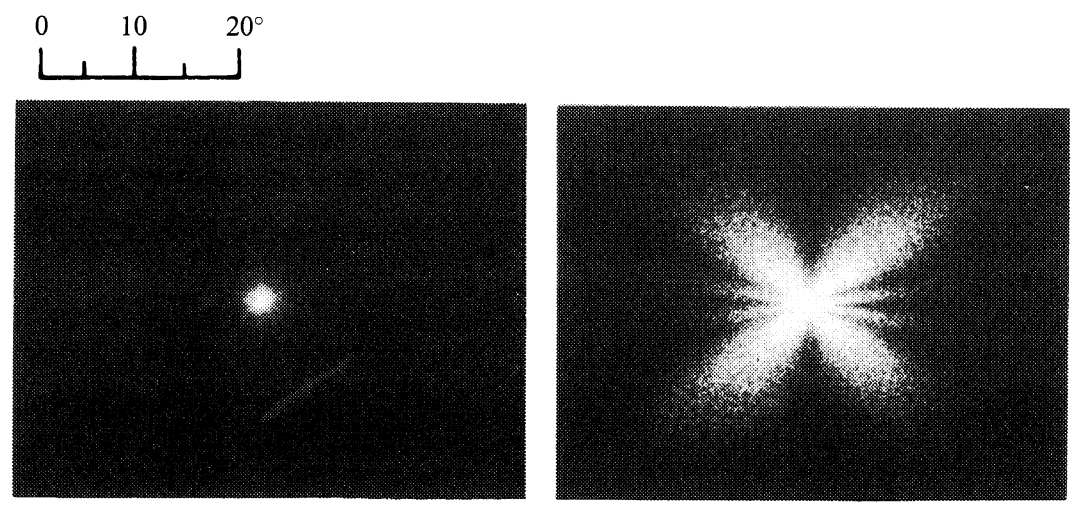

(a) $\lambda=1.0$

(e) $\lambda=2.5$
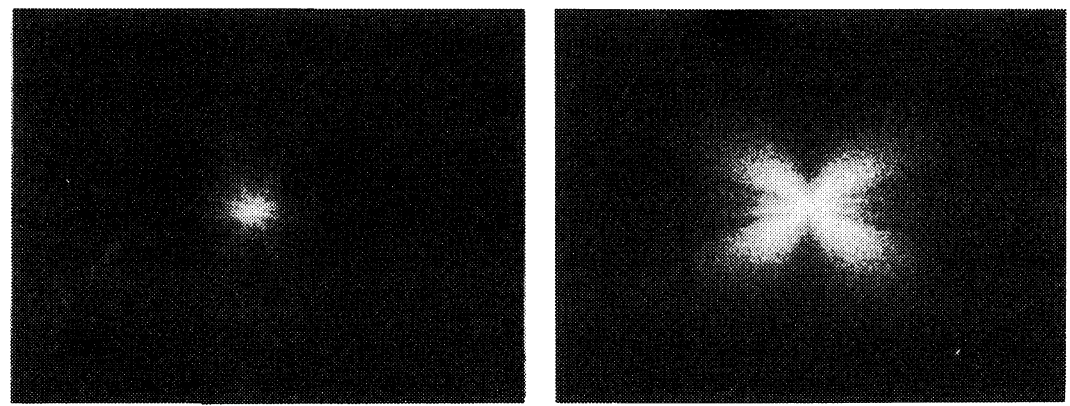

(b) $\lambda=1.2$

(f) $\lambda=3.0$
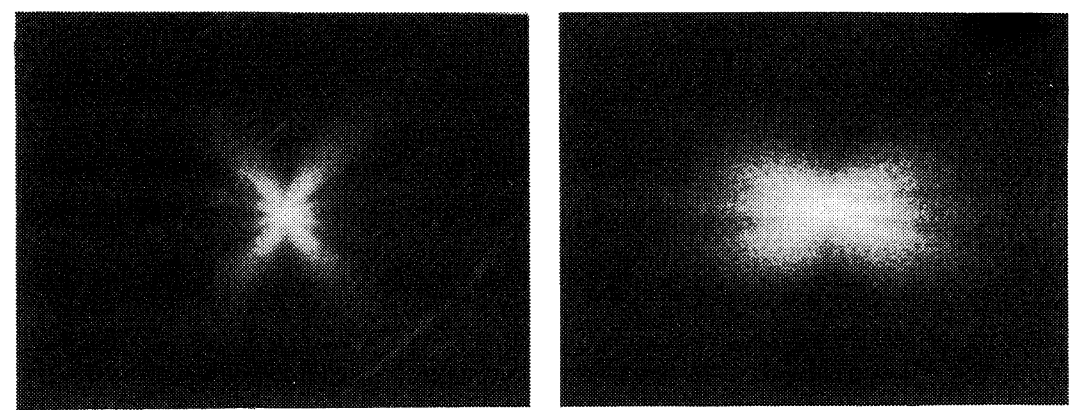

(c) $\lambda=1.5$

(g) $\lambda=3.5$
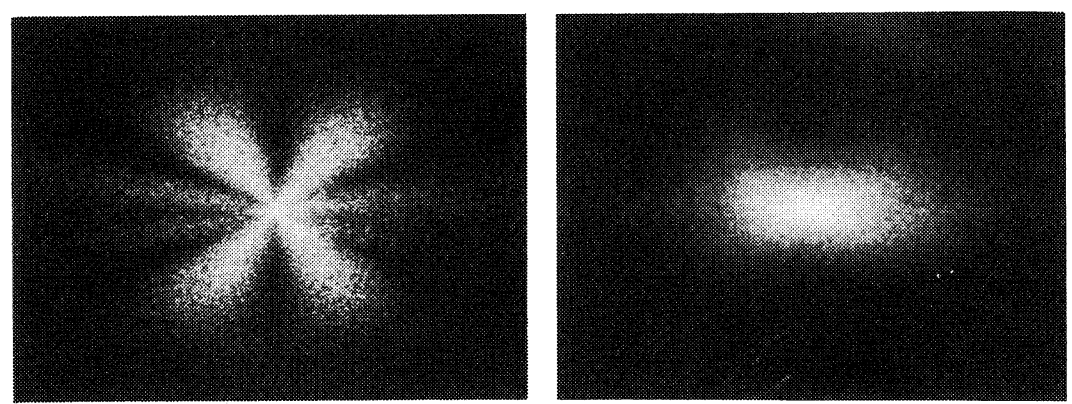

(d) $\lambda=2.0$

(h) $\lambda=4.0$

Figure 11. Change of $H_{\mathrm{v}}$ light scattering patterns of PBT films stretched at $120^{\circ} \mathrm{C}$. 


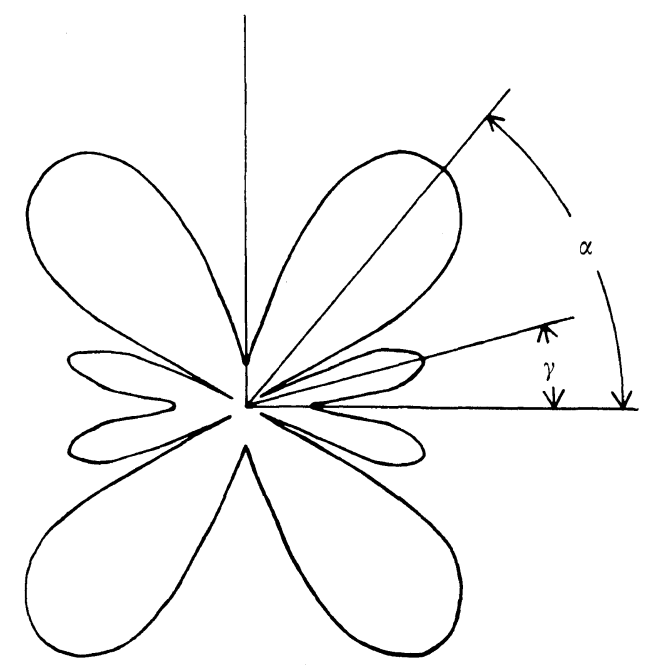

(a)

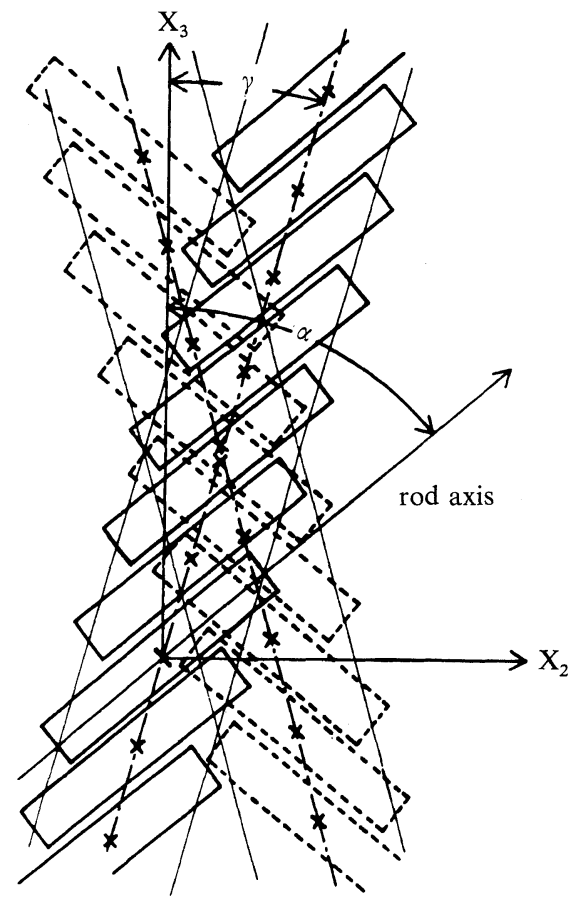

(b)

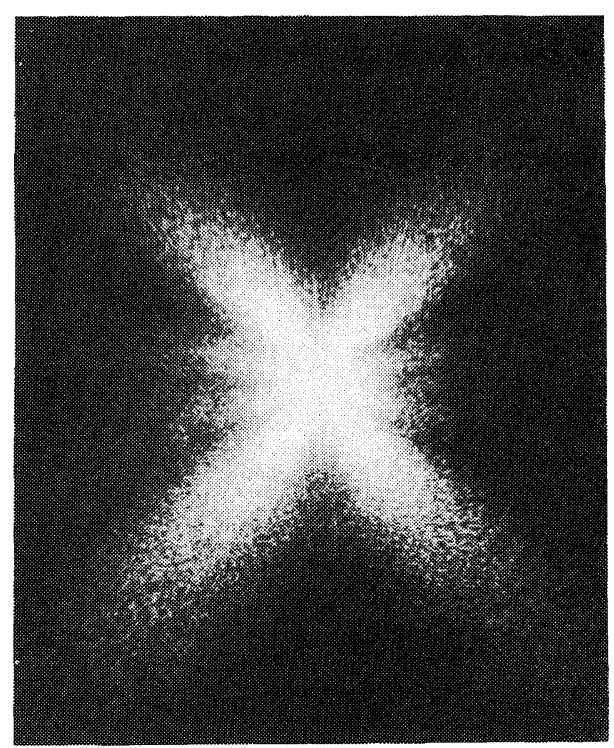

(c)

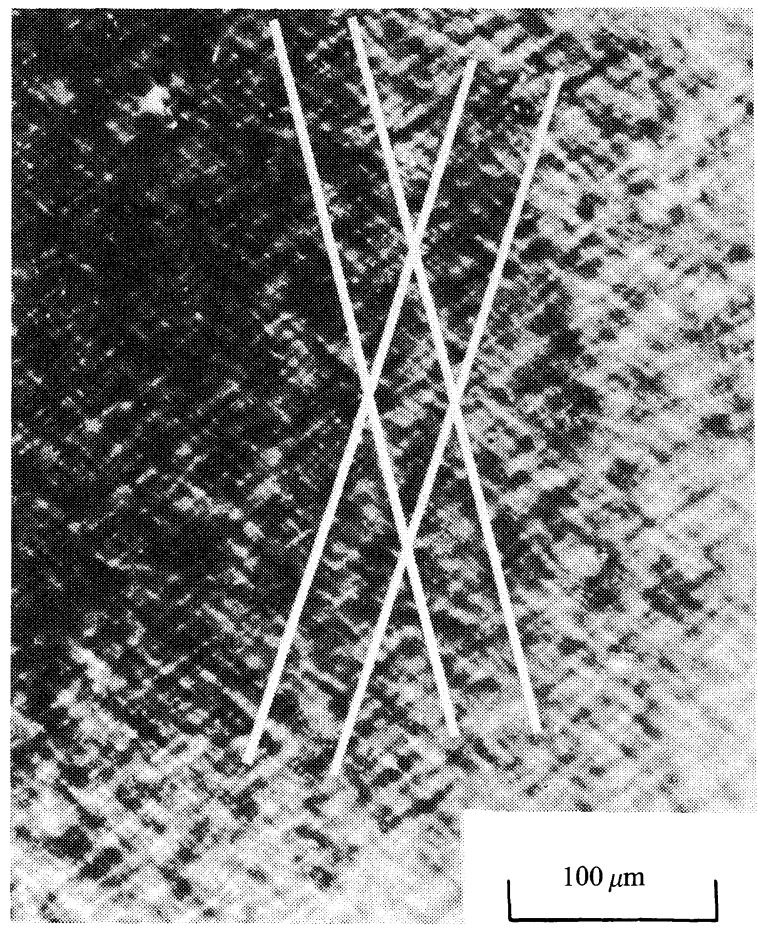

(d)

Figure 12. Schematic diagram for $H_{\mathrm{v}}$ light scattering patterns and oriented rods. (a) schematic representation of $H_{v}$ light scattering pattern; (b) schematic representation of arrangement for rodlike textures with respect to the stretching direction; (c) $H_{\mathrm{v}}$ light scattering pattern observed at $\lambda=1.5$; (d) polarizing micrograph at $\lambda=1.5$. 


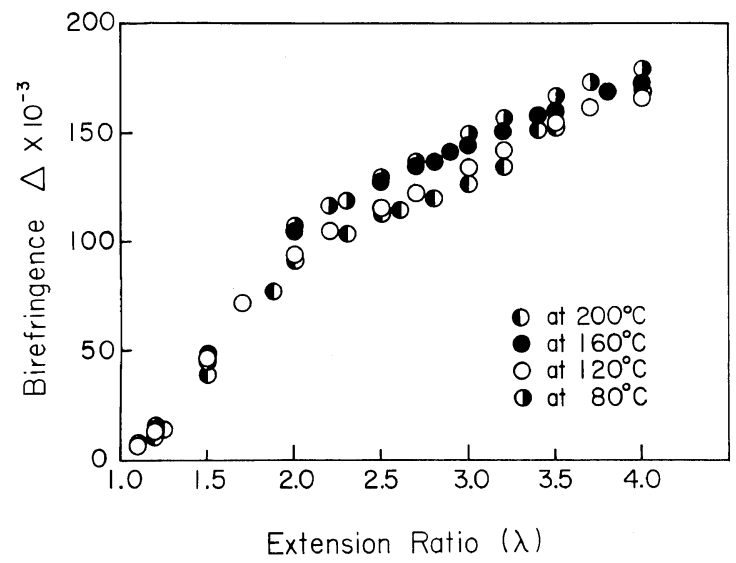

Figure 13. Birefringence $v s$. extension ratio for PBT films stretched at $80,120,160$, and $200^{\circ} \mathrm{C}$.

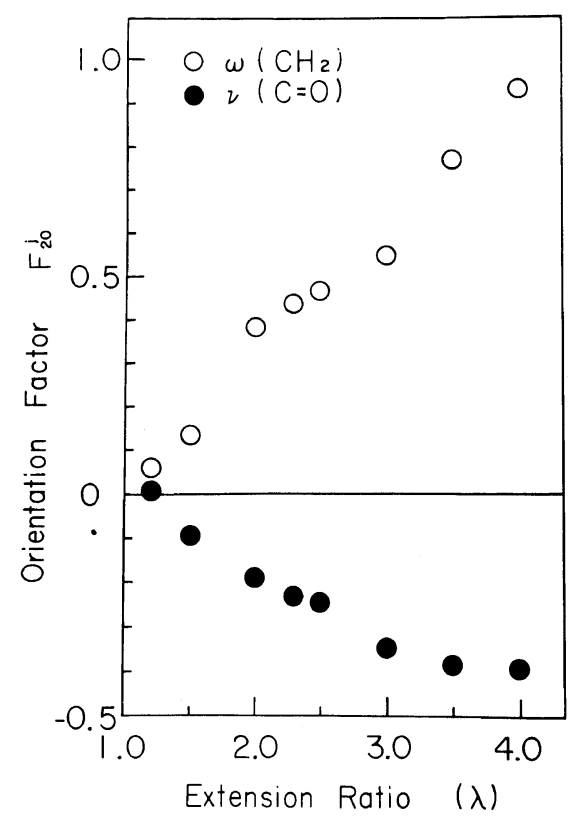

Figure 14. Orientation behavior of PBT molecules in terms of the second order orientation factor of the transition moments of $v(\mathrm{C}=\mathrm{O})$ and $\omega\left(\mathrm{CH}_{2}\right)$.

considerable increase of positive orientation of the PBT molecules with stretching of the films, which is in good agreement with the birefringence results in Figure 13.

Figure 15 shows the changes of the $V_{\mathrm{v}}$ and $H_{\mathrm{h}}$ light scattering patterns of specimens stretched to $\lambda=1.2$ and 2.5 at $160^{\circ} \mathrm{C}$. Generally speaking, the $H_{\mathrm{v}}$ scattering depends upon anisotropy and orientation of the rods, while the $V_{\mathrm{v}}$ and $H_{\mathrm{h}}$ scatterings depend upon density fluctuations between or within the rods and their surrounding medium. Since the scattering arising from the density is isotropic in origin, it therefore does not depend upon a polarization direction. Hence the $V_{\mathrm{v}}$ and $H_{\mathrm{h}}$ scattering patterns are equivalent, if the scattering arises purely from density fluctuations. As shown in Figure 15, the $V_{\mathrm{v}}$ and $H_{\mathrm{h}}$ scattering patterns at $\lambda=$ 1.2 are essentially the same, showing a circular type pattern, although detailed studies show that the $V_{\mathrm{v}}$ scattering intensities have a maximum in the polarization direction perpendicular to the stretching direction. The above result indicates that the density contribution is predominant over the orientation and anisotropy contributions. That is, the density difference between the rods and their surrounding medium are much more effective for the scattering of this film. This indicates that the rods are formed from crystalline regions while the isotropic medium is formed from the amorphous region.

On the other hand, at $\lambda=2.5$, the extension of $V_{\mathrm{v}}$ and $H_{\mathrm{h}}$ scattering lobes becomes remarkable. The difference in these $V_{\mathrm{v}}$ and $H_{\mathrm{h}}$ patterns in comparison with the patterns at $\lambda=1.2$ suggests that the orientation contribution is dominant over the density fluctuation. That is, the $V_{\mathrm{v}}$ pattern is primarily dependent on the anisotropy of the rodlike textures and the orientation distribution of the textures with respect to the stretching direction. The $H_{\mathrm{h}}$ scattering was expected to become a circular pattern indicat- 


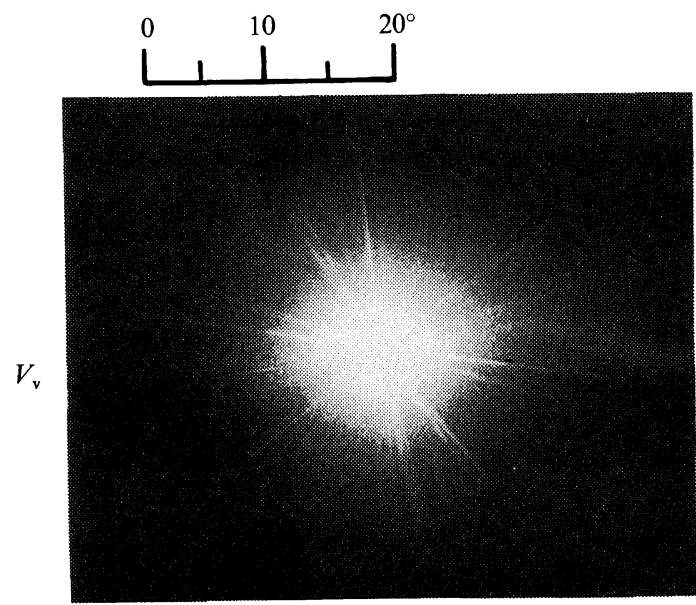

(a) $\lambda=1.2$

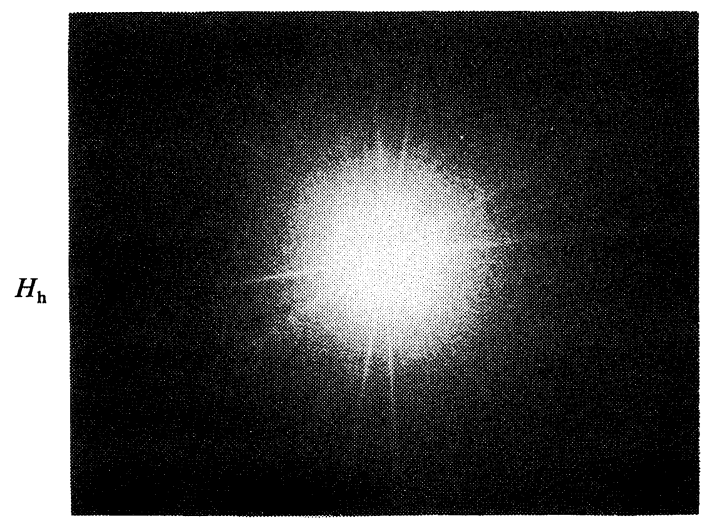

(c) $\lambda=1.2$

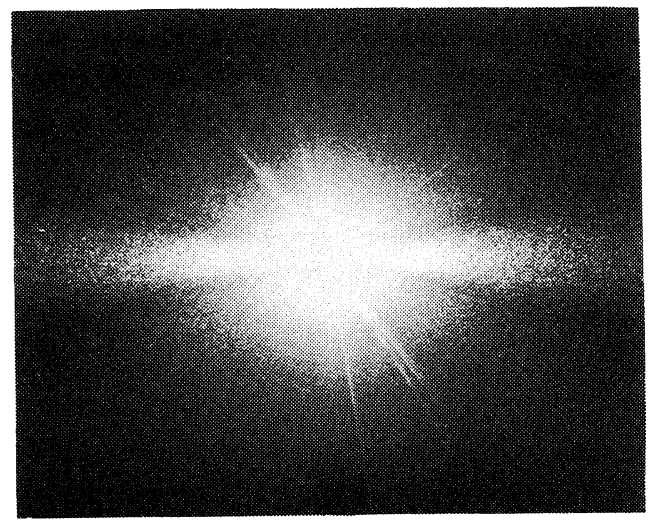

(b) $\lambda=2.5$

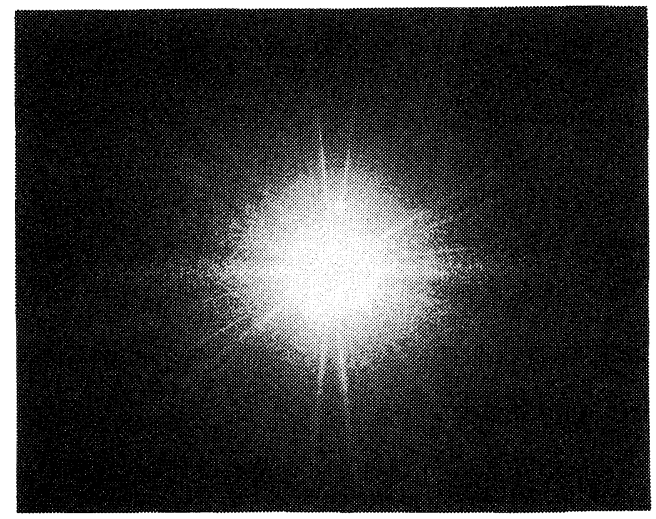

(d) $\lambda=2.5$

Figure 15. $V_{\mathrm{v}}$ and $H_{\mathrm{h}}$ scattering patterns of PBT films at $\lambda=1.2$ and 2.5. The stretching direction is vertical one.

ing the density fluctuation, as the polarization directions of the polarizer and analyzer are perpendicular to the molecular chain axes. The $H_{\mathrm{h}}$ pattern in Figure 15(d) indicates the justice of the above presumption.

\section{CONCLUSIONS}

An interesting phenomenon was found, namely the appearance of rodlike textures of poly(butylene terephthalate) (PBT). The rodlike textures were formed when an amorphous film as well as a film having spherulitic texture was annealed under pressure. This was confirmed using polarizing microscopy and light scattering. Using polarizing micros- copy, it was shown that anisotropic rods showing negative and positive birefringences were oriented randomly. The number of rods showing negative birefringence was more than that showing a positive value. The $H_{\mathrm{v}}$ light scattering pattern is an X-type, showing no indication of intensity maxima along the scattering angle. The intensity was greatest in the scattering center and decreased monotonically with increasing scattering angle. The size of rods in specimen A prepared from the amorphous film is smaller than that in specimen B prepared from the film having a spherulitic texture. When the amorphous film as well as the film having a spherulitic texture were annealed with no pressure, the presence of a rodlike texture was not observed by either 
polarizing microscopy or light scattering. The experimental results mentioned above seem to be important but their significance is still not resolved. In addition, the formation of rodlike textures was found to be essentially independent of an increase of crystallinity.

When the amorphous film with no deformation was preheated for $15 \mathrm{~min}$ at several temperatures between 80 and $200^{\circ} \mathrm{C}$, no superstructure was observed in the specimens obtained, although the crystallinity increased. However, elongation of the specimens yielded rodlike textures oriented at particular angles with respect to the stretching direction forming network unites. At the lower extension ratios such as $\lambda=1.2$, the rods were oriented somewhat predominantly perpendicular to the stretching direction, since the rod axes were oriented at about $\alpha=60^{\circ}$. This indicated the predominant growth of the rods perpendicular to the stretching direction. This mechanism was analyzed in detail by light scattering. The $H_{\mathrm{v}}$ scattering pattern showed a broad four-leaf pattern as lobes and a sharp fourleaf pattern as streaks. In this paper, it was confirmed that the lobes reflect the orientation of rods with respect to the stretching direction and the streaks, which have sometimes been observed for films crystallized from oriented melts, ${ }^{15,16}$ reflect an interparticle interference effect of the rods. The formation of the rods and the orientation behavior were the same for all the specimens stretched at several elongation temperatures between 80 and $200^{\circ} \mathrm{C}$. The results of birefringence and infrared dichroism measurements suggest that PBT molecules are predominantly oriented parallel to the stretching direction with increasing the elongation ratio.

Acknowledgments. The authors are indebted to Professor Cooper of Department of Chemical Engineering, University of Wisconsin, for his valu- able comments and suggestions for the manuscript. Thanks are also due to Professor Kawai, Faculty of Engineering, Kyoto University, and Dr. Ishihara, Katata Institute, Toyo-bo Co., Ltd., for their helpful comments. The authors are also grateful to Dr. R. St. John Manley, Department of Chemistry, McGill University for his kind help with linguistic revisions. The authors also thank Prof. Yasuda and Miss Okuno, Department of Clothing Science, Faculty of Home Economics, Mukogawa Women's University, for their valuable comments and suggestions of DTA measurement.

\section{REFERENCES}

1. W. F. H. Borman and M. Kramer, Am. Chem. Soc. Org. Coat. Plast. Chem. Pap., 34, 77 (1974).

2. R. W. Seymour, J. R. Overton, and L. S. Corley, Macromolecules, 8, 331 (1975).

3. A. Lilaonitkul, J. C. West, and S. L. Cooper, J. Macromol. Sci.-Phys., B12(4), 563 (1976).

4. M. Shen, U. Mehra, M. Niinoma, J. T. Koberstein, and S. L. Cooper, J. Appl. Phys., 45, 4182 (1974).

5. M. Gilbert and F. J. Hybart, Polymer, 13, 327 (1972).

6. M. Gilbert and F. J. Hybart, Polymer, 15, 407 (1974).

7. C. F. Pratt and S. Y. Hobbs, Polymer, 17, 12 (1976).

8. R. S. Stein and A. Misra, J. Polym. Sci., Polym. Phys. Ed., 18, 327 (1980).

9. S. Clough, J. J. van Aartsen, and R. S. Stein, J. Appl. Phys., 36, 3072 (1965).

10. E. J. Roche, R. S. Stein, and E. L. Thomas, J. Polym. Sci., Polym. Phys. Ed., 18, 1145 (1980).

11. C. A. Boye, Jr. and J. R. Overton, Bull. Am. Phys. Soc., 19, 352 (1974).

12. M. B. Rhodes and R. S. Stein, J. Polym. Sci., A-2, 7, 1539 (1969).

13. T. Hashimoto, K. Nagatoshi, and H. Kawai, Polymer, 17, 1075 (1976).

14. M. Matsuo, F. Ozaki, H. Kurita, S. Sugawara, and T. Ogita, Macromolecules, 13, 1187 (1980).

15. T. Hashimoto, A. Todo, and H. Kawai, Polym. J., 10, 521 (1978).

16. M. Matsuo, M. Tamada, T. Terada, C. Sawatari, and M. Niwa, Macromolecules, 15, 985 (1982). 